\title{
SMOLTIFICATION AND BREEDING FOR RAPID GROWTH MAY SUPPRESS IMMUNITY OF ATLANTIC SALMON: EVIDENCE FROM TRANSCRIPTOME ANALYSES
}

\author{
Aleksei Krasnov $^{1 \S}$, Sergey Afanasyev ${ }^{l, 2}$, Matthew Baranski $^{l}$, Maria Dahle $^{3}$, Lill-Heidi Johansson ${ }^{l}$, \\ Sven Martin Jørgensen ${ }^{1}$, Simon Mackenzie ${ }^{5}$, Nick Robinson ${ }^{l}$, Espen Rimstad ${ }^{4}$, Gerrit Timmerhaus ${ }^{l}$ \\ ${ }^{1}$ Nofima AS, Norwegian Institutes of Food, Fisheries \& Aquaculture Research, Ås, Norway \\ ${ }^{2}$ Sechenov Institute of Evolutionary Physiology and Biochemistry, Saint Petersburg 194223, Russia \\ ${ }^{3}$ Section of Immunology, Norwegian Veterinary Institute, Oslo, Norway \\ ${ }^{4}$ Department of Food Safety and Infection Biology, Norwegian University of Life Sciences, Oslo, \\ Norway
}

The first months after seawater transfer (SWT) of fish are a period of heavy losses in commercial farming of Atlantic salmon. A large part of the mortality is due to infectious diseases, but unsuccessful smoltification and various undetected pathogens may contribute to cases that remain unexplained. SWT is preceded with smoltification, which is associated with dramatic endocrine and metabolic changes. Farmed Atlantic salmon has spent many generations under intense selective breeding for rapid growth and other commercially important traits. Given the high energy costs of smoltification and growth one may anticipate a trade off between these processes and immunity. We report studies that used gene expression profiling with oligonucleotide microarrays and RNA-seq. (1) Smoltification was induced with photoperiod manipulation, and microarray analyses were performed in samples from the head kidney, gill and intestine. Massive down-regulation of immune genes was revealed, starting from the middle of stimulation and remained constant to the end of experiment - three weeks after SWT. The innate antiviral immunity showed greatest suppression. The only deviation from the common trend was a transient increase of Ig transcripts in the gill shortly after SWT. (2) RNA-seq analyses in hearts of farmed parr and smolts produced similar results: all functional groups of immunity were down-regulated while expression of Ig increased. All immune genes in hearts of wild salmon showed higher expression in comparison with farmed fish. (3) Parr and smolts from the same batch reared respectively in fresh and seawater were challenged with PRV, the causative agent of HSMI. Microarray analyses found a minor difference between the uninfected fish. Further, parr developed more rapid responses to the pathogen, as observed by earlier induction of innate antiviral and B cells responses in the head kidney and to a lesser extent in the spleen. Clearance of PRV from spleen was more effective in parr. (4) Microarray analyses found slight but significant up-regulation in several groups of immune genes in the gill of wild anadromous salmon during smoltification, which was in contrast to farmed salmon. In conclusion, results obtained in several independent experiments, including different research stations, wild and commercial fish of different origin, provided indications for compromised immune competence. It remains unknown however, whether down-regulation of immune genes affects resistance of Atlantic salmon to pathogens and if recovery takes place in seawater. It is also important to figure out if immune suppression is linked to and a consequence of breeding for rapid growth.

Key words: Atlantic salmon, IgM, variable region, NGS, natural antibodies

${ }^{\S}$ Corresponding author. Telephone: +47 64970484. E-mail address: Aleksei.Krasnov@nofima.no 